\title{
Reflets
}

Revue ontaroise d'intervention sociale et communautaire

\section{L’étranger : ogre ou bon Samaritain?}

\section{Nico Trocmé}

Volume 2, numéro 1, printemps 1996

Contrer la violence subie par les femmes et les enfants en milieu familial

URI : https://id.erudit.org/iderudit/026105ar

DOI : https://doi.org/10.7202/026105ar

Aller au sommaire du numéro

Éditeur(s)

Reflets : Revue ontaroise d'intervention sociale et communautaire

ISSN

1203-4576 (imprimé)

1712-8498 (numérique)

Découvrir la revue

Citer cet article

Trocmé, N. (1996). L'étranger : ogre ou bon Samaritain? Reflets, 2(1), 58-68. https://doi.org/10.7202/026105ar

\section{Résumé de l'article}

Les programmes de prévention s'adressant aux enfants, concernant le risque d'enlèvement oud'agression sexuellle ou phsique, reposent sur des efforts de prévention face à l'étranger. Laréalité des enfants est toute autre. L'auteur montre que la maltraitance physique et sexuelle enOntario est le fait de personnes connues de l'enfant, tout particulièrement des parents.
Tous droits réservés ㄷ Reflets : Revue ontaroise d'intervention sociale et communautaire, 1996
Ce document est protégé par la loi sur le droit d'auteur. L'utilisation des services d'Érudit (y compris la reproduction) est assujettie à sa politique d'utilisation que vous pouvez consulter en ligne.

https://apropos.erudit.org/fr/usagers/politique-dutilisation/ 


\section{L'étranger : ogre ou bon Samaritain?}

L es programmes de prévention s'adressant aux enfants, concernant le risque d'enlèvement ou d'agression sexuellle ou phsique, reposent sur des efforts de prévention face à l'étranger. $L$ a réalité des enfants est toute autre. L 'auteur montre que la maltraitance physique et sexuelle en 0 ntario est le fait de personnes connues de l'enfant, tout particalièrement des parents.

\section{NicoTrocmé}

P rofesseur, U niversité de Toronto

\section{Introduction}

M on fils est revenu de l'école le jour de l'H alloween ayant appris qu'il devait demander à ses parents d'inspecter ses bonbons pour prévenir tout risque d'empoisonnement. C e soir là, nous sommes allés de maison hantée en maison hantée quêter des bonbons, avons participé à une des fêtes communautai res les plus importantes de l'année. Q uelle leçon doit-on tirer de ces événements? Voisins, étrangers, monstres, bonbons, poisons? Lestraditionscommunautaires complexes de I'H alloween représentent bien notre attitude confuse envers l'étranger.

L'étranger a toujours joué un rôle ambigu dans l'éducation de nos enfants. Alors que les contes de Perrault le présentent souvent comme sorcière ou ogre, dévoreur d'enfants, les contes d'A ndersen le projettent bien plus souvent dans le rôle de sage ou de bon Samaritain. Ce double message se reflète également dans les messages contradictoires que nous donnons à nos enfants: «sois poli avec la dame», «dis bonjour au monsieur», «pourquoi ne vas-tu pas jouer avec cette petite fille?», «n'accepte pas les bonbons des étrangers», «ne va jamais dans la voiture d'un étranger».

C ette confusion apparente présente généralement l'étranger en tant qu'inconnu, c'est-à-dire une personne à connaître par 
«... les enfants courent moins de risques de subir de telles situations aux mains des étrangers qu'aux mains de leurs propres familles»
I'intermédiaire des parents. A lors que les enfants apprennent éventuellement à naviguer dans cette société complexe de grandes personnes, il arrive de temps en temps, malheureusement, que l'étranger passe du rôle de l'inconnu à celui de l'ogre. II suffit d'une rumeur d'enlèvement d'enfant pour éveiller en nous les histoires d'ogres, de loups, et de sorcières. N ous transmettons alors à nos enfants un message moins subtil: prends garde, car derrière l'étranger souriant peut se cacher un loup; plus il est gentil, plus il faut se méfier. Ce glissement de sens est dangereux, car il risque de susciter chez l'enfant de la méfiance qui, loin de le protéger de l'ogre, l'empêche de faire la connaissance d'un voisin et d'accepter l'aide du bon samaritain.

$Q$ uels sont les vrais risques? Faut-il enseigner aux enfants la méfiance ou la confiance? U n examen de l'incidence des enlèvements d'enfants et des agressions sexuelles et physiques contre les enfants, montre que les enfants courent moins de risques de subir de telles situations aux mains des étrangers qu'aux mains de leur propre famille. De plus, une revue des programmes de prévention montre que beaucoup d'enfants, en particulier les enfants en bas âge, comprennent mal les messages de prévention. Ces réalités indiquent que, plutôt que leur apprendre à se méfier desogres, la plusimportante leçon de prévention est d'encourager les enfants à accepter l'aide des bons Samaritains.

\section{Enlèvements d'enfants}

La télévision, les journaux, les affiches de métro, et même les panneaux publicitaires de camions nous montrent, de façon régulière, des photos d'enfants disparus. Q ui sont-ils? O nt-ils été enlevés par des étrangers? Joan Fisher (1989), analyste au bureau du Solliciteur général du $C$ anada, a fait une étude des enfants disparus rapportés à la police. L'analyse a été faite à partir d'un échantillon de 12446 cas officiels d'enfants disparus, rapportés en 1986 dans trois centres urbains du Canada, et représentant $29 \%$ de la population nationale. C omme l'indique le tableau 
ci-dessous, moins de $3 \%$ des enfants portés disparus étaient vicitmes d'enlèvement, et seulement 9 cas sur 12446 étaient enregistrés sous la catégorie «enlèvement par un étranger», soit $0,07 \%$ des enfants disparus. U ne analyste du Bureau d'enregistrement des enfants disparus, $M$ arlene $D$ aley, a fait une analyse nationale des signalements d'enfants disparus(D aley, 1995). Q uatre vingt-dix-neuf cas présumés d'enlèvement par des étrangers ont été signalés à la police en 1988. D ans $97 \%$ de ces cas, les enfants ont été retrouvés et n'avaient pas été enlevés par un étranger. Seuls trois cas se sont révélés être de véritables enlèvements par des étrangers, et les trois enfants ont tous été retrouvés sains et saufs.

Tableau 1 - Types d'enfants disparus dans trois centres urbains du $C$ anada

Type de cas nombre de cas pourcentage

\section{A Enlèvement}

étranger

9

parent gardien

parent non-gardien

membre de famille

autre

Total des enlèvements

\section{B Fuite}

institution

foyer d'accueil

parent

Total des fuites

\section{Autre}

perdu

retrouvé, non-reporté

autre

Total autre

Grand total

source: (Fisher, 1989)
4024

7840

5832

10696
234

7

10

323

\section{1}

178

868

1427

12446

3

$1 \%$

$2 \%$

$3 \%$

$32 \%$

$7 \%$

$47 \%$

$86 \%$

$3 \%$

$1 \%$

$7 \%$

$11 \%$

$100 \%$ 
Aux États-U nis, Joel Best (1990) note que le nombre d'enfants enlevés varie considérablement selon la source d'information. II donne comme exemple les taux rapportés en 1984, qui varient de 69 cas officiellement enquêtés par la police fédérale (FBI) jusqu'à 50000 cas (Estimation du $\mathrm{N}$ ational $\mathrm{C}$ entre for $\mathrm{M}$ issing and Exploited $C$ hildren). Best estime que le nombre véritable d'enfants enlevés par des étrangers est de l'ordre de 500 caspar année. D avid Finkelhor et ses collègues ont essayé d'examiner le phénomène de plus près à partir d'un sondage téléphonique de 10326 parents choisis au hasard (Finkelhor et al., 1995b). À leur suprise, ils n'ont pas été capables d'identifier un seul cas d'enlèvement d'enfant par un étranger. En revanche, ils ont identifié 35 cas où le parent interrogé soupçonnait qu'un étranger avait essayé d'enlever son enfant. II faut noter que, dans $80 \%$ de ces cas, aucune force n'a été utilisée, l'enfant a été blessé dans un seul cas, et la police a été contactée dans $42 \%$ des cas. L'analyse du profil de ces familles indique que, dans un bon nombre de situations, il est possible que l'interprétation des parents ait été influencée par leur propreexpérience de traumatismesenfantins(Finkel hor et al., 1995b).

\section{Maltraitance physique et sexuelle en Ontario}

Les données de l'É tude ontarienne de l' incidence et des caractérisitiques des enfants maltraités (EOI: Trocmé, M cPhee, $\mathrm{H}$ ay, 1994) permettent de comparer l'incidence des actes commis par des parents ou autres personnes connues des enfants, par rapport aux étrangers. C esdonnées proviennent d'un échantillon aléatoire de 2447 enquêtes menées par les Sociétés d'aide à l'enfance de l'O ntario. L'étude a classé les résultats d'enquêtes en trois catégories: enquêtes confirmées ( $27 \%$ ), enquêtes présumées (30\%), et enquêtes non-fondées (43\%). Les enquêtes étaient classées en fonction de quatre formes de maltraitance: sévices, agression sexuelle, négligence, et maltraitance émotionelle. Les cas de sévices et d'agression sexuelle constituaient près de $60 \%$ des enquêtes. Les enfants étaient de tous âges, avec une forte représentation d'adolescents. 
«... il est dair que les membres de la famille et les gens connus de la famille posent une menace bien plus sérieuse que les étrangers. »
Le second tableau présente lestypes d'agresseurs dans les cas confirmés d'agression sexuelle et de sévices. Les parents étaient responsables de plus de $90 \%$ des cas de sévices, et d'autres membres de la famille étaient responsables de $5 \%$ des cas. Dans les cas d'agression sexuelle, nous constatons immédiatement une distribution très différente. Les parents, surtout les pères, sont tenus responsables dans $20 \%$ des cas. En grande majorité, les agresseurs sont néanmoins des personnes connues de l'enfant (80 \%): autres membres de la famille, amis, connaissances. Dix pour cent des agresseurs tombent dans la catégorie «nconnu», qui comprend à la fois de véritables étrangers, et des personnes connues de l'enfant mais qui n'ont pu être identifiées. II faut également noter que le risque d'agression par un étranger est plus faible dans le cas de jeunes enfants que pour les adolescents. Sans aucun doute ces situations sont tragiques, mais il est clair que les membres de la famille et les gens connus de la famille posent une menace bien plus sérieuse que les étrangers.

Tableau 2 - A gresseurs identifiés dans les cas confirmés ou présumés de sévices et d'agression sexuelle confirmés ou suspectés, selon l'É tude ontarienne d' indidence de $1993(N=820)$

\begin{tabular}{|c|c|c|c|c|}
\hline & \multicolumn{2}{|c|}{ Sévices } & \multicolumn{2}{|c|}{ Agression sexuelle } \\
\hline & $\begin{array}{c}\% \\
\text { de cas } \\
\end{array}$ & $\begin{array}{c}\text { N ombre } \\
\text { de cas }\end{array}$ & $\begin{array}{c}\% \\
\text { de cas } \\
\end{array}$ & $\begin{array}{c}\text { Nombre } \\
\text { de cas }\end{array}$ \\
\hline Père & $49 \%$ & 4527 & $23 \%$ & 1528 \\
\hline Beau père/ partenaire & $10 \%$ & 930 & $10 \%$ & 689 \\
\hline M ère & $43 \%$ & 4011 & $3 \%$ & 162 \\
\hline Frère & $1 \%$ & 125 & $12 \%$ & 781 \\
\hline Autre membre & & & & \\
\hline $\begin{array}{l}\text { de la famille } \\
\text { A mi, voisin ou }\end{array}$ & $4 \%$ & 338 & $19 \%$ & 1226 \\
\hline autre connaissance & e $3 \%$ & 266 & $22 \%$ & 1471 \\
\hline Inconnu & $2 \%$ & 170 & $11 \%$ & 734 \\
\hline
\end{tabular}

II faut noter que les données de l'Eo I ne représentent que les cas rapportés aux Sociétés d'aide à l'enfance, et excluent certains cas cas rapportés à la police, notament certains cas de maltraitance 
«... les politiques et pratiques d'intervention fondées sur des situations atypiques et sensationelles finissent par obliger les Sodétés de protection de I'enfance à concenter leurs ressources aux enquêtes et programmes de dépistage, les empêchant de faire du travail de base avec les familles en diffiaulté.» aux mains des étrangers. U ne analyse des cas signalés à la police, que nous sommes en train d'effectuer, indique que la proportion de cas de sévices et d'agression sexuelle impliquant des étrangers est plus grande, surtout parmi les adolescents. Pourtant, dans les cas documentés par la police, les membres de la famille et autres connaissances constituent un plus grand risque pour les enfants que les étrangers (Trocmé et Brison, en cours).

Le contraste entre nos perceptions du problème des enlèvements d'enfants et la réalité a poussé Best (1990) à faire une analyse des réactions du public à l'égard du phénomène des enfants disparus. Best (1990) considère les motivations qui incitent les médias et les organisations comme le $\mathrm{N}$ ational $\mathrm{C}$ entre for $\mathrm{M}$ issing and $\mathrm{Exploited} C$ hildren à user de sensationalisme et d'exagération dans le traitement des enfants kidnappés. Son analyse situe le problème en partie sur le plan de la compétition sur le «marché des problèmes sociauX». Le concept de l'enfant kidnappé par un étranger est un problème social à forte teneur émotionnelle, et qui a servi à élaborer des législations sur la protection de l'enfance, ou plus récemment, des programmes de prévention de la violence contre les enfants. En Angleterre, Parton a documenté le même phénomène dans certains cas tragiques de meurtres d'enfant. Son analyse se fait autour du concept de «panique morale» qui définit la nature du travail des agences de protection. Selon Best et Parton, les politiques et pratiques d'interventions fondées sur des situations atypiques et sensationelles, finissent par obliger les Sociétés de protection de l'enfance à concentrer leurs ressources sur les enquêtes et programmes de dépistage, les empêchant de faire du travail de base avec les familles en difficulté. Trop souvent, les programmes élaborés dans la panique morale finissent par échouer lorsque sont révélées de fausses promesses sur les possibilités de protection.

\section{Efficacité des programmes de prévention}

Les précautions envers les étrangers que nous essayons d'enseigner 
à nos enfants sont non seulement problématiques à cause de la nature du message enseigné, mais posent également des problèmes au niveau de la capacité des enfants de comprendre et d'intégrer ces messages. Les évaluations de programmes scolaires de prévention des agressions sexuelles fournissent un bon exemple de la complexité de ce genre de travail. En général, ces programmes montrent que la majorité des enfants exposés à ces programmes retiennent des leçons importantes, notamment celle de demander de l'aide. Pourtant, ce succès est plus mitigé auprès des jeunes enfants. Gil Berrick et N eil G ilbert (1991) ont évalué en C alifornie huit programmes de prévention pour enfants de première et troisième année. En moyenne, ces enfants ont réussi à accroître de $11 \%$ le pourcentage de réponses correctes, sur un questionnaire distribué au début et à la fin du programme. $M$ ais une analyse détaillée des «progrès» indique une certaine confusion chez les enfants de première année. En particulier, ces enfants ont de la difficulté à comprendre les distinctions plus nuancées. Leurs réponses aux questions sur le toucher et sur les étrangers indiquent une tendance à croire que toutes les formes de contact physique sont mauvaises, et que seuls les étrangers posent un risque.

D avid Finkelhor et Jennifer D ziuba-L eatherman (1995a) ont fait aux États-U nis, une enquête sur un échantillon aléatoire de 2000 enfants et de leurs parents pour estimer l'impact des programmes de prévention. Ils ont trouvé que deux tiers et plus des enfants avaient été exposésà au moins un programme de prévention. Alors qu'en majorité, les parents et les enfants avaient une attitude positive envers ces programmes, le sondage a montré que dans le cas de jeunes enfants, les parents rapportaient des niveaux plus élevés d'angoisse et de peur ( $23 \%$ des enfants en cinquième année avaient plus peur des adultesà cause des messages de prévention). De plus, les résultats n'ont pas réussi à montrer d'effets positifs concrets. Les taux de victimisation étaient aussi élevés chez les enfants ayant suivi les programmes de prévention que chez les enfants ne l'ayant pas suivi (D aro, 1994)

Les résultats ambigus de ces programmes $n$ 'indiquent pas que les efforts de prévention avec les enfants jeunes sont inutiles. Les programmes qui cherchent à enseigner des stratégies précises de 
«Il faudrait pouvoir apprendre aux jeunes enfants à distinguer entre un contad affectueux et un contact sexuel, à savoir quand obér et quand désobéir un adulte, et à comprendre que tous les adultes, y compris ses propres parents, posent un certain risque. « protection et utilisent des techniques de rôle, ont généralement mieux réussi (D aro, 1994). Par exemple, Fryer et M iyoshi (1987a) montrent le succès d'un programme créé spécialement pour apprendre aux enfants du jardin d'enfants et de première année à ne pas quitter l'école avec un étranger. À la fin du programme, chaque enfant a été accosté par un étranger qui l'a invité à quitter l'école. Dix-huit des 23 enfants du groupe expérimental ont refusé de suivre l'étranger, alors que 11 des 21 enfants du groupe de contrôle ont fait de même. La reprise du test six mois plus tard a produit les mêmes résultats (Fryer et M iyoshi, 1987b).

La réussite du programme de prévention étudié par Fryer et M iyoshi (1987a, 1987b) montre qu'il est possible d'enseigner des comportements préventifs aux jeunes enfants, mais seulement dans un contexte étroitement défini. D e tels programmes seraient justifiables si les dangers d'abus étaient seulement limités aux étrangers approchant les enfants dans les cours d'école. Comme les statistiques discutées ci-dessus le montrent, les situations qui posent des risquesaux enfants sont bien plus complexes. II faudrait pouvoir apprendre aux jeunes enfantsà distinguer entre un contact affectueux et un contact sexuel, à savoir quand obéir et quand désobéir à un adulte, et à comprendre que tous les adultes, y compris ses propres parents, posent un certain risque. Et même sil était possible de le faire, voudrions-nous vraiment enseigner de telles leçons à des enfants de cinq, six et sept ans? ? $^{1}$

\section{Conclusion}

L'instinct protecteur est fondamental chez les parentset éducateurs.

R econnaissant le développment inéluctable des enfants, nous apprenons à modifier cet instinct suffisamment pour permettre à nos enfants de prendre les risques nécessai res. Le développement des enfants exige que nous apprenions à maintenir cet équilibre, qui devient plus difficile plus l'enfant grandit. Q uand un enfant de douze mois apprend à marcher, le parent doit évaluer le risque d'une chute en fonction du niveau de développment de l'enfant. 
«L es risques de sévices et d'agression sexuel sont beaucoup plus élevés quand les enfants sont avec des gens connus à qui I'on fait confiance, que lorsqu'ils sont avec des étrangers. »
C ette analyse se fait relativement facilement, mais devient plus complexe au fur et à mesure que l'enfant passe du milieu protégé de la famille au monde de l'école et du quartier. Avec cette transition, nous passons des risques visibles à des risques invisibles difficilement pondérables. Parfois, notre instinct protecteur évalue mal ces risques. Par exemple, de plus en plus de parents préfèrent conduire leurs enfants à l'école plutôt que de les laisser aller à pied sans adulte qui les accompagne. $C$ ette décision minimise les risques d'accidents de voiture et surestime les risques d'agression par des étrangers.

La protection des enfants doit, autant que possible, se fonder sur une évaluation objective des risques. Les étrangers posent peu de risques aux enfants. Les enlèvements d'enfants par des étrangers sont extrêmement rares. Les risques de sévices et d'agression sexuelle sont beaucoup plus élevés quand les enfants sont avec des gens connus, à qui l'on fait confiance, que lorsqu'ils sont avec des étrangers. La présentation de l'étranger comme ogre potentiel n'est pasjustifiée. C'est non seulement une présentation inexacte des risques véritables, mais la peur de l'étranger risque de décourager la demande d'aide, tout en créant une fausse sécurité avec les adultes familiers.

Souvent, les jeunes enfants n'ont pas la capacité de comprendre les messages complexes de protection que nous essayons de leur enseigner. En revanche, ils comprennent bien les offres d'aide. Au terme de leur évaluation des programmes scolaires de prévention des agressions sexuelles, Finkelhor et Strapko (1992) concluent que le succès principal de ces programmes est d'identifier les enfants maltraités. Alors qu'il y a peu de preuves que les enfants apprennent à mieux se défendre contre les agresseurs, sont ces programmes toujours suivis d'une augmentation du nombre d'enfants qui révèlent avoir été victimes d'abus. L'importance de demander de l'aide est un message que les enfants peuvent comprendre. Plutôt que d'enseigner la méfiance, il faut chercher à enseigner la confiance. Les enfants doivent être encouragés à se tourner vers toutes les formes d'aide possibles, qu'elles viennent des parents, des enseignants ou même des étrangers. 


\section{Note}

1. Trois mois après la fête d'H alloween, mon fils de six ans est revenu de l'école après avoir appris «trois façons de frapper les gens». II venait de participer à un programme de prévention des agressions CAP-ESPACE. J'ai participé quelques semaines plus tard à un atelier CAP-ESPACE pour adultes. Le programme m'a impressionné. U tilisant des jeux de rôle centrés sur trois situations d'agression: agression entre enfants, agression par un adulte inconnu, agression par un adulte connu, le programme cherche à aider les enfants à développer trois stratégies de prévention: affirmation de soi, soutien des pairs, et confiances à un adulte. C es leçons ont eu peu d'effet sur mon fils. II se souvenait bien des deux dames et du monsieur qui sont venus en classe, il se souvenait très bien des trois façons de frapper (affirmation de soi?), mais le reste de la leçon lui a complètment échappé.

\section{Bibliographie}

BER R ICK, J. D., et N. GILBERT (1991). W ith the best of intentions: The sexual abuse prevention movement. $\mathrm{N}$ ew York, Guilford Press.

BEST, J. (1990). Threatened children: R hetoric and concern about child-victims. Chicago, U niversity of Chicago Press.

DALEY, M. L. (1995). M issing children's registry. R C M P G az ette, Vol. 51, no 12, 10-19.

DAR O, D. (1994). Prevention of C hild Sexual A buse. T he F uture of C hildren, Vol.4, no 2, 198-223.

FIN KELHO R, D. (1994). Current Information on the Scope and N ature of Child Sexual A buse. The F uture of $C$ hildren, Vol. 4 no 2, 31-53.

FIN KELHOR, D., et N. STR APKO (1992). Sexual abuse prevention education. In D. Willis, W. $\mathrm{H}$ olden, et M . R osenberg ( $E d$.), Prevention of child maltreatment: $D$ evelopmental and ecological perspectives. N ew York, John W iley et Sons.

FIN KELHOR, D., et J. DZIU BA-LEATHER M AN (1995a).V ictimization prevention programs: A national survey of children's exposure and reactions. C hild A buse and N eglect, Vol.19 no 2, 129-139.

FIN KELHOR, D., G. HOTALIN G, et N. ASIGIAN (1995b). Attempted nonfamily abductions. C hild Welfare, Vol. 74 no 5, 941-958.

FISHER , J. (1989). M issing children research project V olume I: F indings of the study. 0 ttawa, Solicitor General $C$ anada.

FRYER , G. E., S. K. KR AIZER, et T. MIYOSHI (1987). M easuring actual reduction of risk to child abuse: A new approach. C hild A buse and N eglect, Vol. 11, 173-179. 
FRYER , S. K., et T. MIYOSHI (1987). M easuring children's retention of skills to resist stranger abduction - $U$ se of the simulation technique. $C$ hild $A$ buse and $N$ eglect, Vol. 11 no 2, 181-185.

TROCMÉ, N., et R. BRISON (en cours). Assaults and homicides. In Anonymous, Injuries to children. $O$ ttawa, Statistics $C$ anada.

TR OCMÉ, N., D. M CPHEE, K. K.TAM et T. HAY, (1994). 0 ntario incidence study of reported child abuse and negled, Toronto, Institute for the Prevention of $C$ hild $A$ buse. 